\title{
Does lung allocation score maximize survival benefit from lung transplantation?
}

\author{
Mark J. Russo, MD, MS, ${ }^{a}$ Berhane Worku, MD, ${ }^{\mathrm{b}}$ Alexander Iribarne, MD, MS, ${ }^{\mathrm{b}}$ \\ Kimberly N. Hong, MHSA, ${ }^{\mathrm{b}}$ Jonathan A. Yang, MD, ${ }^{\mathrm{b}}$ Wickii Vigneswaran, MD, ${ }^{\mathrm{a}}$ and \\ Joshua R. Sonett, MD
}

\begin{abstract}
Objective: The lung allocation score was initiated in May 2005 to allocate lungs on the basis of medical urgency and posttransplant survival. However, the relationship between lung allocation score and candidate outcomes remains poorly characterized. The purpose of this study was (1) to describe outcomes by lung allocation score at the time of listing and (2) to estimate the net survival benefit of transplantation by lung allocation score.
\end{abstract}

\begin{abstract}
Methods: The United Network for Organ Sharing provided de-identified patient-level data. Analysis included lung transplant candidates aged 12 years or more and listed between May 4, 2005, and May 4, $2009(\mathrm{n}=6082)$. Candidates were stratified according to lung allocation score at listing into 7 groups: lung allocation score less than 40,40 to 49,50 to 59, 60 to 69,70 to 79,80 to 89 , and 90 or more. Outcomes of interest included the risk of death on the waiting list and likelihood of transplantation. The net survival benefit of transplantation was defined as actuarial median posttransplant graft survival minus actuarial median waiting list survival, where the outcome of interest was death on the waiting list or posttransplant; candidates were censored at the time of transplant or last follow-up.
\end{abstract}

Results: In the lowest-priority strata (eg, $<40$ and $40-49$ ), less than $4 \%$ of candidates died on the waiting list within 90 days of listing. The median net survival benefit was lowest in the lung allocation score less than 40 ( -0.7 years) and lung allocation score $90+$ group (1.95 years) and highest in the 50 to 59 (3.44 years), 60 to 69 (3.49 years), and 70 to 79 (2.81 years) groups.

Conclusions: The mid-priority groups (eg, 50-59, 60-69, 70-79) seem to achieve the greatest survival benefit from transplantation. Although low-priority candidates comprise the majority of transplant recipients, survival benefit in this group seems to be less than in other groups given the low risk of death on the waiting list. As expected, both the time to transplant and survival on the waitlist are lower in the higher-priority strata (eg, 80-89 and 90+). However, their net survival benefit was likewise relatively low as a result of their poor posttransplant survival. (J Thorac Cardiovasc Surg 2011;141:1270-7)

The disparity between potential recipients and available donors demands efficient methods of organ allocation to ensure optimal use of this scarce resource. In prior years, lung allocation was based on accrued time on the waiting list. ${ }^{1-3}$ In 1999, the US Department of Health and Human

From the Section of Cardiac and Thoracic Surgery, ${ }^{\mathrm{a}}$ University of Chicago Medical Center, Chicago, Ill; and the Division of Cardiothoracic Surgery, ${ }^{\mathrm{b}}$ Department of Surgery, College of Physicians and Surgeons, Columbia University, New York, NY This work was supported in part by Health Resources and Services Administration contract 231-00-0115 and the National Institutes of Health training Grant 5T32HL007854-13 (to Drs Iribarne and Yang). The views expressed in this article are those of the authors alone and do not necessarily reflect the views or policies of the Department of Health and Human Services or the National Institutes of Health, nor does the mention of trade names, commercial products, or organizations imply endorsement by the US Government.

Disclosures: Authors have nothing to disclose with regard to commercial support.

Read at the 37th Annual Meeting of The Western Thoracic Surgical Association, Ojai, California, June 23-26, 2010.

Received for publication June 19, 2010; revisions received Dec 4, 2010; accepted for publication Dec 20, 2010.

Address for reprints: Mark J. Russo, MD, MS, Section of Cardiac and Thoracic Surgery, University of Chicago Medical Center, 5841 S. Maryland Avenue, MC 5040, Chicago, IL 60637 (E-mail: mrusso@surgery.bsd.uchicago.edu).

$0022-5223 / \$ 36.00$

Copyright (c) 2011 by The American Association for Thoracic Surgery doi:10.1016/j.jtcvs.2010.12.028
Services published the "Final Rule," which required that all organ allocation systems place less emphasis on waiting time and more on medical urgency. ${ }^{4}$ In response to the "Final Rule," the organ procurement and transplantation network and the United Network for Organ Sharing (UNOS) implemented the lung allocation score (LAS) in May 2005. Under LAS, all lung transplant candidates are prioritized according to LAS, which is calculated on the basis of a multivariate model that is a weighted combination of predicted waitlist and posttransplant survival at 1 year (Appendix 1). ${ }^{1}$

Several studies, including those by our group, have demonstrated favorable trends in waitlist times and waitlist survival since the implementation of the LAS. ${ }^{5-7} \mathrm{An}$ increase in disease severity among listed patients has also been observed. ${ }^{5,6,8}$ Despite this trend, acceptable posttransplant survivals have been demonstrated in the LAS era. ${ }^{5-7,10}$ However, as previously demonstrated, as expected, posttransplant survival is inversely related to LAS at the time of transplant. ${ }^{11,12}$

The objectives of this study were (1) to describe outcomes by LAS at the time of listing and (2) to estimate 

Abbreviations and Acronyms
LAS = lung allocation score
LAS-L $=$ LAS at listing
LAS-T $=$ LAS at transplant
UNOS $=$ United Network for Organ Sharing

the net survival benefit of transplantation by LAS score. It has been suggested that a higher LAS score corresponds not only to an earlier likelihood of transplantation but also to a greater net transplant benefit. ${ }^{1,13,14}$ However, because LAS weighs expected waiting list survival more heavily than expected posttransplant survival, it is possible that an increase in posttransplant morbidity and mortality may result in less overall benefit. This may be especially true at the highest LAS scores. To date, the relationship between LAS and candidate outcomes remains poorly characterized, and no studies have analyzed the overall transplant benefit associated with various LAS ranges.

\section{MATERIALS AND METHODS Data Collection}

Use of data in this analysis is consistent with the regulations of our university's institutional review board and the UNOS Data Use Agreement. The Standard Transplant Analysis and Research Dataset were provided by UNOS (data source 020909-3). The dataset contains information collected from the UNetsm database forms, including the Transplant Candidate Registration form, Transplant Recipient Registration form, and Transplant Recipient Follow-up form. These data are the basis for the UNOS Thoracic Registry.

\section{Study Population}

The UNOS provided de-identified patient-level data for all lung transplant candidates and recipients in the United States. Analysis included lung transplant candidates aged 12 years or more and listed between May 4, 2005, and May 4, $2009(\mathrm{n}=6082)$. Follow-up data were provided through November 19, 2009. Patients were followed from the date of listing until death, transplantation, or date of last known follow-up, and from the date of transplantation until death, retransplantation, or date of last known follow-up, which was the last day of follow-up data provided by UNOS. Recipients who underwent simultaneous transplantation of another organ $(n=10)$ and those with missing LAS data $(\mathrm{n}=53)$ were excluded from the analysis.
To estimate survival on the waiting list across various ranges of LAS, lung transplant candidates were stratified into 7 groups according to LAS at listing (LAS-L): LAS-L less than 40,40 to 49,50 to 59,60 to 69,70 to 79,80 to 89 , and 90 or greater. To estimate posttransplant survival across various ranges of LAS, lung transplant recipients were stratified into 7 groups according to LAS at transplant (LAS-T): LAS-T less than 40, 40 to 49,50 to 59,60 to 69,70 to 79,80 to 89 , and 90 or greater. For simplicity, candidates with LAS less than 40 and 40 to 49 were referred to as "low-priority"; candidates with LAS 50 to 59, 60 to 69 , and 70 to 79 were referred to as "mid-priority"; and candidates with LAS 80 to 89 and $90+$ were referred to as "higher-priority."

\section{Outcome Measures}

Survival analysis. For waiting list survival, candidates were followed from date of initial listing to death on the waiting list, transplant, or last follow-up. The outcome of interest was death on the waiting list; candidates were censored at the time of transplant or as lost/alive at last known follow-up.

For posttransplant graft survival, recipients were followed from date of transplant to graft failure (defined by patient death or retransplantation) or last known follow-up. The outcome of interest was graft loss; candidates were censored as lost/alive at last known follow-up. $\triangle$ LAS was defined as LAS at the time of transplant minus LAS at the time of listing.

Net survival benefit of transplantation. Net survival benefit was defined as actuarial posttransplant graft survival minus actuarial survival on the waiting list at various time points (including 90 days post-listing vs 90 days posttransplant, 1 year post-listing vs 1 year posttransplant, and 2 years post-listing vs 2 years posttransplant).

The median net survival benefit of transplantation was defined as actuarial median posttransplant graft survival minus actuarial median survival on the waiting list.

\section{Data Analysis}

All data were analyzed using the statistical software package Stata 9 (Stata Corp, College Station, Tex). Continuous variables were reported as means, and categoric variables were reported as frequencies. Continuous variables were compared with the Student $t$ test, and categoric variables were compared with the chi-square test. All reported $P$ values are 2-sided.

\section{RESULTS}

\section{Patient Characteristics}

LAS strata, including mean LAS-L \pm standard deviation, LAS-T \pm standard deviation (mean and median), $\triangle$ LAS (mean and median), and wait times are summarized in Table 1.

TABLE 1. Lung allocation score strata

\begin{tabular}{|c|c|c|c|c|c|c|c|c|c|c|c|c|c|c|c|}
\hline \multirow[b]{2}{*}{ LAS } & \multicolumn{4}{|c|}{ LAS at listing } & \multicolumn{5}{|c|}{ LAS at transplant } & \multicolumn{3}{|c|}{$\triangle \mathrm{LAS}$} & \multicolumn{3}{|c|}{ Wait time (d) } \\
\hline & $\mathbf{n}$ & $\%$ & Mean & SD & $\mathbf{n}$ & $\%$ & Mean & SD & Median & Mean & SD & Median & Mean & SD & Median \\
\hline$<40$ & 4796 & $69.9 \%$ & 34.1 & 2.6 & 3251 & $68.4 \%$ & 38.8 & 11.7 & 35.3 & 4.65 & 11.2 & 0.52 & 267.0 & 311.4 & 149.5 \\
\hline $40-49$ & 1260 & $18.4 \%$ & 43.9 & 2.7 & 1002 & $21.1 \%$ & 51.1 & 14.3 & 45.8 & 7.26 & 13.9 & 0.06 & 102.0 & 144.4 & 47.0 \\
\hline $50-59$ & 305 & $4.4 \%$ & 54.2 & 2.9 & 224 & $4.7 \%$ & 61.8 & 13.3 & 57.1 & 7.60 & 13.1 & 0.03 & 58.3 & 85.9 & 26.0 \\
\hline $60-69$ & 145 & $2.1 \%$ & 64.3 & 2.9 & 99 & $2.1 \%$ & 70.5 & 13.4 & 66.6 & 6.23 & 13.3 & 0.04 & 45.9 & 81.0 & 21.0 \\
\hline 70-79 & 97 & $1.4 \%$ & 74.8 & 2.9 & 63 & $1.3 \%$ & 76.7 & 10.6 & 75.8 & 1.94 & 10.3 & 0.02 & 43.5 & 108.5 & 15.0 \\
\hline $80-89$ & 154 & $2.2 \%$ & 85.8 & 3.1 & 69 & $1.5 \%$ & 84.0 & 10.4 & 87.5 & -1.76 & 10.0 & 0.00 & 36.9 & 93.9 & 9.0 \\
\hline $90+$ & 105 & $1.5 \%$ & 92.2 & 1.4 & 47 & $1.0 \%$ & 90.6 & 6.8 & 92.1 & -1.60 & 6.6 & 0.00 & 27.8 & 89.1 & 7.0 \\
\hline Total & 6862 & & 40.0 & 12.7 & 4755 & & 45.3 & 17.1 & & 5.18 & 11.84 & & 58.3 & 252.79 & \\
\hline
\end{tabular}

LAS, Lung allocation score; $S D$, standard deviation. Shows mean LAS-L \pm standard deviation, LAS-T \pm standard deviation (mean and median), $\triangle \mathrm{LAS}$ (mean and median), and wait times. 


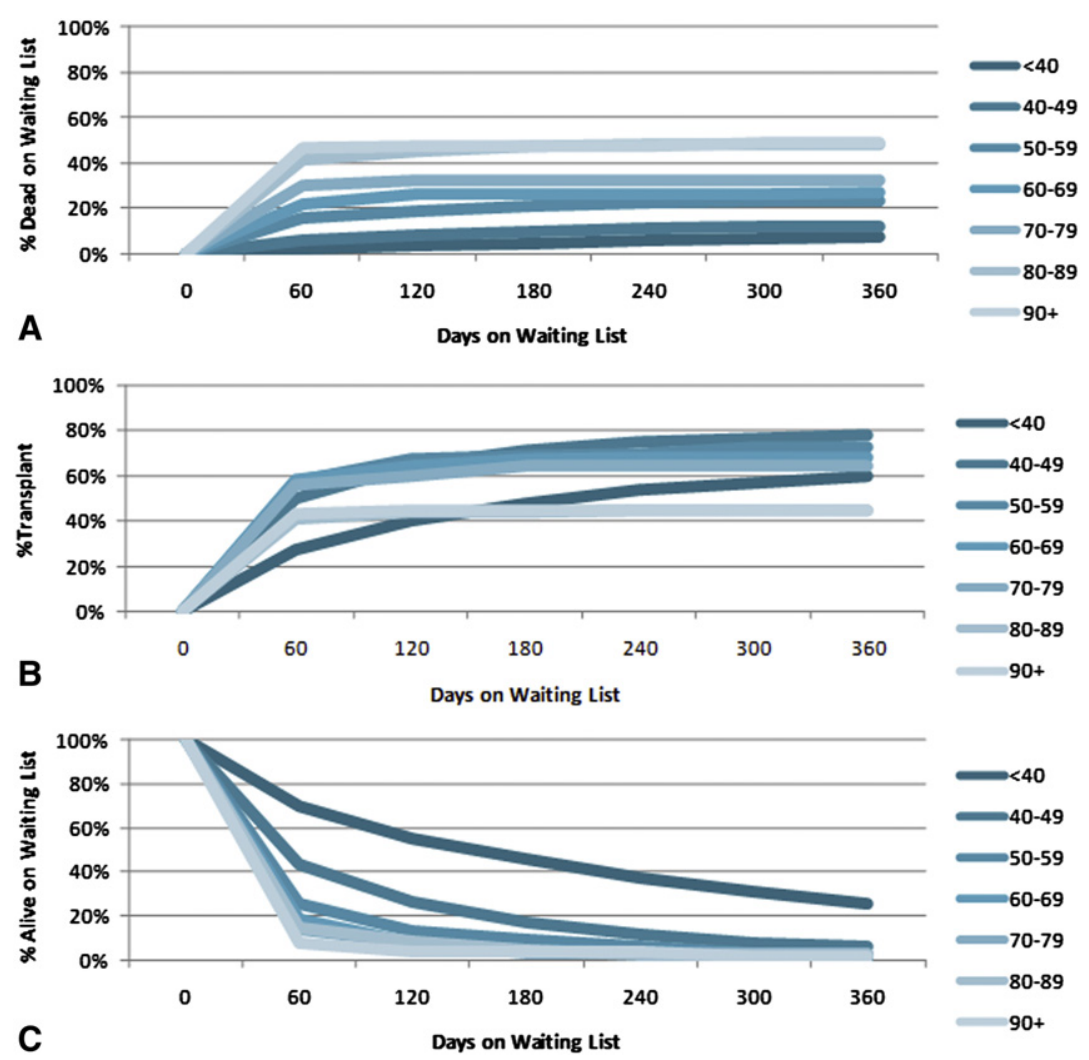

FIGURE 1. Waitlist outcomes, including the percentage of candidates, stratified by LAS-L, who (A) died on the waiting list, (B) underwent transplantation, and $(\mathrm{C})$ were alive on the waiting list as a function of the number of days accrued on the waiting list.

\section{Waitlist Outcomes}

Figure 1 summarizes waitlist outcomes, including the percentage of candidates, stratified by LAS-L, who died on the waiting list (A), underwent transplantation (B), and were alive on the waiting list as a function of the number of days accrued on the waiting list (C). With the exception of the LAS-L less than 40 group, few candidates $(1 \%-6 \%)$ were alive on the waiting list at 1 year. Candidates were lost from the waiting list because of transplantation or death according to LAS-L score. Waitlist mortality increased in a stepwise fashion for all LAS-L strata. At 1 year, $45 \%$ of candidates in the higher-priority strata (80-89 and 90+) underwent transplantation and $49 \%$ were dead on the waiting list, whereas only $1 \%$ remained alive on the waiting list. In the lower-priority strata (4049 ), at 1 year $77 \%$ of patients underwent transplantation and $12 \%$ were dead on the waiting list, whereas only $6 \%$ remained alive on the waiting list. In the lowest priority stratum $(<40)$, both waiting list mortality $(7 \%)$ and transplantation rates $(60 \%)$ were the lowest, and as a result, the number of candidates still alive on the waiting list was the highest $(26 \%)$. The middle-priority strata (5070) had the greatest proportion of candidates undergoing transplantation.

\section{Net Transplant Benefit}

Figure 2 demonstrates median posttransplant survival stratified by LAS-T, median waitlist survival by LAS-L, and the median net survival benefit of transplantation by LAS-T. The net survival benefit was lowest in the LAS less than 40 ( -0.7 years) and LAS $90+(1.95$ years) groups and highest in the 50 to 59 (3.44 years), 60 to 69 (3.49 years), and 70 to 79 (2.81 years) groups.

Figure 3 demonstrates that from listing to transplant there was little movement between strata. The horizontal axis represents strata at listing, and the bars represent LAS-T. Figure 3 shows that $86.9 \%$ of candidates in the lowpriority group remained in the low-priority group at the time of transplant. Likewise $75.7 \%$ of the mediumpriority group and $92.9 \%$ of the high-priority group remained in their respective groups at transplant.

\section{DISCUSSION}

Before LAS, lung allocation was based on time accrued on the waiting list. ${ }^{1-3}$ Although time on the waiting list was intended to be a surrogate for disease severity, it likely led to practices that had the opposite effect. Patients were listed earlier in the course of their disease to accrue more time, and those who made it to the top of the 


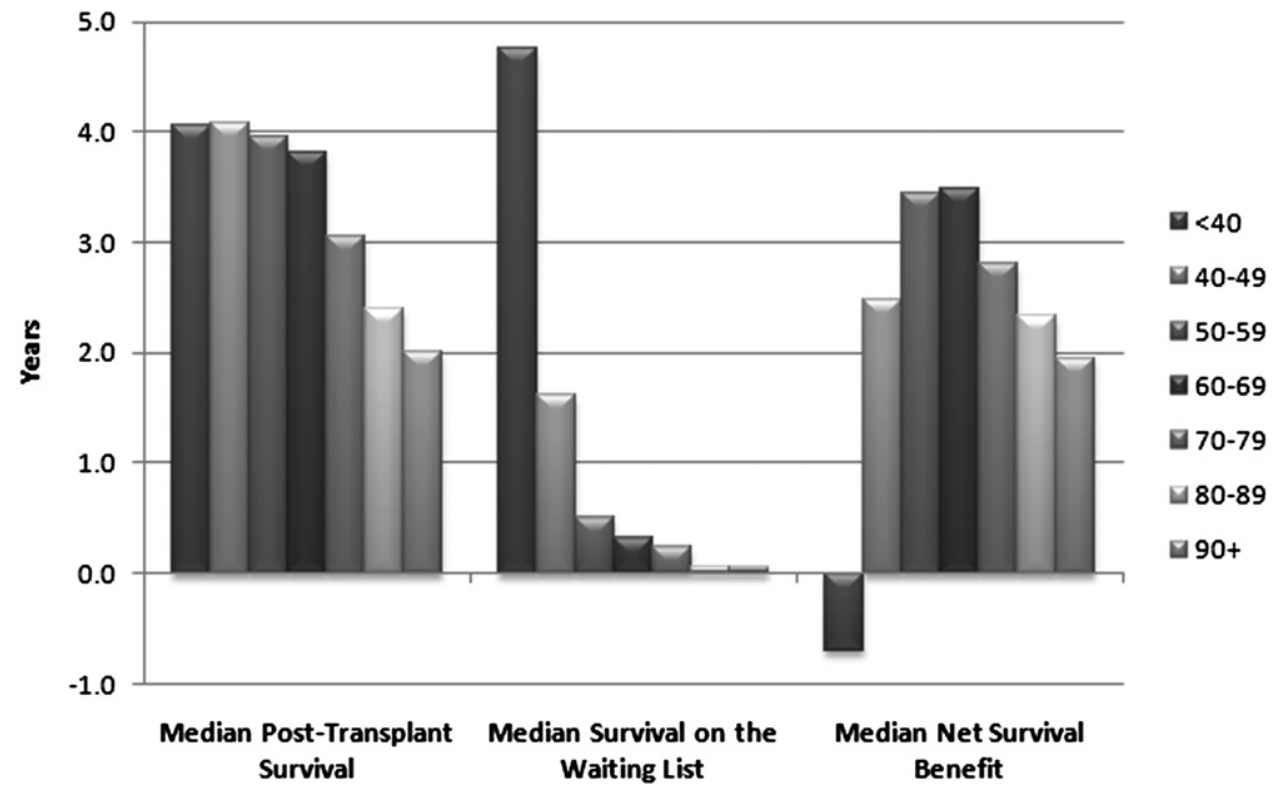

FIGURE 2. Median posttransplant survival stratified by LAS-T, median waitlist survival by LAS-L, and the median net survival benefit of transplantation by LAS-T.

list may have been healthy enough to wait longer. ${ }^{3,12,13}$ Conversely, the sickest patients, who had greater urgency, may not have been listed because of the perception that they would have little chance of surviving the wait for a donor organ. ${ }^{5}$ The goal of the LAS is to eliminate these potential biases and further maximize transplant benefit per organ by simultaneously minimizing waitlist mortality while maximizing posttransplant survival. The LAS uses a variety of clinical factors determined from multivariable modeling to estimate a patient's likelihood of survival at 1 year on the waitlist and posttransplant. ${ }^{1}$

When the LAS was first introduced, critics expressed concern that because LAS placed greater weight to expected waitlist survival than posttransplant survival, this system may compromise posttransplant outcomes by preferentially allocating organs to more critically ill patients. Subsequently, however, a number of studies have demonstrated no adverse effects on waitlist time and mortality ${ }^{5-8}$

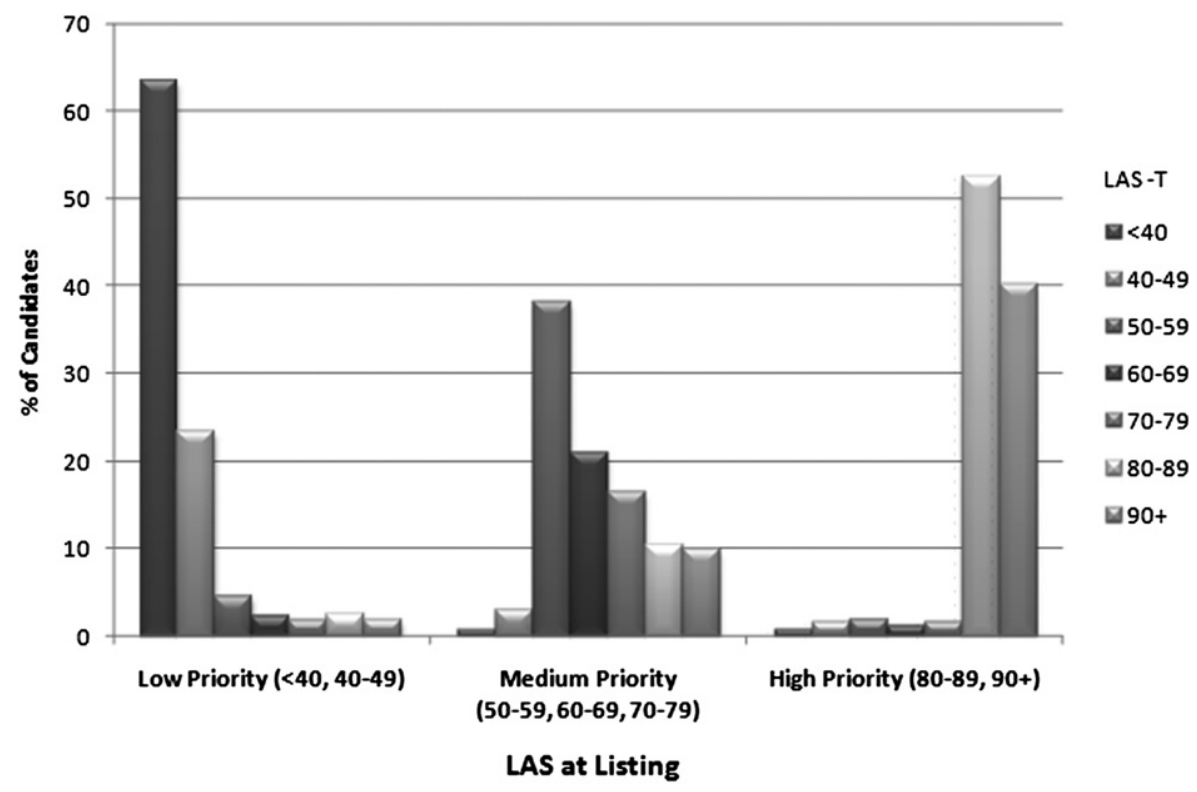

FIGURE 3. LAS at transplant by LAS at listing. 
or early posttransplant mortality, ${ }^{5,7-9}$ despite the increased acuity of transplant recipients. ${ }^{5,6,8}$ Nevertheless, in subgroup analysis, recipients with higher LAS scores were shown to have higher rates of postoperative complications, lengths of stay, and posttransplant short- and long-term mortality. $^{7,10,11}$ These studies analyzed posttransplant survival, but no studies have estimated the net benefit of transplantation.

\section{Outcomes on the Waiting List}

The results of this current study demonstrate that LAS score is associated with waitlist outcomes. The outcome of interest (death on the waiting list or transplantation) was determined in most candidates within the first year. Among candidates with LAS-L $40+$, few $(1 \%-6 \%)$ were alive on the waiting list at 1 year. Also, among candidates with LAS-L $60+$, less than $10 \%$ were alive on the waiting list at 90 days. As expected, the proportion of patients who died on the waiting list increased in a stepwise manner as LAS-L increased. Although a relatively small proportion of candidates in the lower LAS-L strata died within 1 year, approximately one half of the high-priority candidates died on the waiting list within 1 year.

The proportion of candidates undergoing transplantation demonstrated a more complex relationship. The lower and mid-priority groups demonstrated higher rates of transplantation than the lowest or highest groups. In the highest strata, the incidence of transplantation decreased because waitlist mortality was high. In fact, a larger proportion of candidates in the LAS-L less than 40 group underwent transplantation than in the high-priority groups (eg, LAS-L 80-90 and 90+).

\section{Net Survival Benefit}

This analysis further suggests that the lowest priority ( $<40$ and 40-49) and highest priority (80-90 and 90+) LAS groups had the lowest survival benefit. Surprisingly, given the relatively low risk of death on the waiting list, candidates with LAS less than 40 seem to have better survival in the absence of transplantation, whereas candidates with LAS 40 to 49 gain limited survival benefit from transplantation. In the high-priority LAS groups (80-89 and 90+), a significantly higher percentage of patients were alive 1 year posttransplant compared with 1 year post-listing in the absence of transplantation. Nevertheless, although survival on the waiting list in the absence of transplantation is poor, posttransplant survival is also poor. Thus, although LAS effectively ranks lung transplant candidates on the basis of degree of illness, the highest scores do not translate into the greatest survival benefit. This discordance between LAS score and survival benefit is likely due to the greater weight of waitlist survival in the LAS algorithm.

It should be noted that estimates of survival on the waiting list, and therefore the net survival benefit of transplanta- tion, face limitations. This survival analysis assumes candidates were censored randomly. However, this assumption is not entirely true because this analysis censored candidates at the time of transplant, which is not a random event. Under the LAS, sicker patients receive preference in organ allocation. Nevertheless, this limitation may not significantly influence the findings presented here for 2 reasons. First, the LAS-T, for most recipients, was not significantly different than LAS-L (Figure 3). Furthermore, in the highest LAS groups, the median posttransplant survival is so limited it has minimal impact on the calculated net survival. That is, given median posttransplant survival in the $90+$ group is 2 years, the calculated net benefit will be low regardless of expected waitlist survival. Therefore, this potential for confounding is unlikely to change the conclusions of this analysis.

\section{Implications}

Findings from the current analysis have a number of important implications. First, although LAS was developed to preferentially allocate organs to the highest priority candidates, the majority of organs, approximately 9 of every 10 , were allocated to lower-priority candidates. This is particularly troublesome given the findings from this analysis that limited survival benefit is offered by low-priority candidates receiving transplants, and a significant proportion of high-priority candidates with greater potential for benefit die waiting for an organ. This analysis also raises questions regarding the highest LAS group receiving transplants given their poor posttransplant survival. Although shortterm survival (at 90 days and 1 year posttransplant) seems to be improved with transplantation in this group, longerterm survival seems to be limited.

In an effort to avoid inefficient allocation of scarce organs and truly maximize the potential benefit from available organs, additional modifications to the current allocation system should be considered. First, further study is needed to better understand why such a large number of organs are allocated to lung transplant recipients in the low-priority group, whereas a significant number of candidates in the higher-priority groups die on the waitlist. This may result from preferential allocation of organs to local, thus relatively lower-priority, recipients. Second to prevent allocation of organs to extremely high-risk candidates, modification of the LAS calculation should be considered in an effort to truly maximize net benefit from transplantation. We do not believe that centers should be strictly prohibited from performing transplantation in any specific candidate. However, as we have discussed, we support modifications to the LAS that give lower priority to candidates expected to have a poor outcome and higher priority to candidates likely to receive greater benefit. ${ }^{15}$ It is possible that this may be accomplished by placing less emphasis on expected waiting list survival and more on expected posttransplant 
survival. Alternatively, development of a posttransplant risk stratification score could be imposed to discourage candidates with an expected posttransplant mortality exceeding a predefined threshold from receiving a transplant, and used to inactivate the candidates or decrease their LAS. Another strategy is to use an alternate listing system, which is already in use in other organ types, including heart transplantation. Under such a system, high-risk recipients are only eligible for organs that are not accepted for another candidate and will otherwise be discarded. Such a system maximizes the transplant benefit of optimal organs, but can run the risk of further diminishing outcomes by pairing the sickest recipients with marginal organs.

\section{Study Limitations}

These data have several limitations. First, large patient registries may often suffer from incompleteness in data entry. Fields contained within this database, however, were generally well populated with a $90 \%$ to $99 \%$ data entry rate for the majority of variables. Patients with missing data were not included in the analysis; however, given the large sample size, it is unlikely that these excluded patients would have significantly altered the results. Second, despite the fact that the UNOS reporting system provided definitions for variables in data guidelines, there could be inaccuracies in individual center reporting to UNOS. However, the major outcome variables in this study, such as LAS, waitlist time, waitlist mortality, and number of transplant candidates listed, are major variables used in patient and center evaluation, and would thus likely receive careful attention in reporting. Third, because the LAS was developed in 2005, these data should be considered short-term and will require further analysis for assessment of longitudinal trends. Additional follow-up is needed, including longerterm outcomes, such as 3- and 5-year posttransplant survival. Fourth, LAS and other strategies for predicting posttransplant outcomes have been shown to be only mildly predictive of posttransplant outcomes. ${ }^{16}$ Finally, this analysis considers only survival benefit; it does not consider quality of life or economic measures. Although the UNOS provides functional status data on recipients posttransplant, it is generally poorly populated, and there are no longitudinal quality of life data on waiting list candidates. Findings from this analysis highlight the importance of these data, especially given the finding of limited survival benefit in the lower-priority strata. It is possible that despite the limited survival benefit in some subgroups, there may be a significant benefit in terms of qualityadjusted life years. Therefore, a national effort should be implemented to measure quality of life in candidates and recipients alike. Furthermore, future studies should assess candidates' preferences, including their willingness to trade long-term survival for improved quality of life. This may be particularly relevant to candidates in the lower-priority strata who have longer survival on the waiting list, but poor quality of life.

\section{CONCLUSIONS}

Increasing LAS is associated with increasing likelihood of death on the waiting list. With the exception of the LAS less than 40 group, few candidates $(1 \%-6 \%)$ were alive on the waiting list at 1 year. Low-priority candidates (eg, <40 and 40-49) comprise the majority of transplant candidates and recipients. Net survival benefit in lowpriority candidates seems to be less than mid-priority groups, given the low risk of waiting list death. Midpriority strata (eg, 50-59, 60-69, 70-79) seem to have the highest rate of transplantation at 1 year (along with 40-49) and the greatest survival benefit from transplantation. In the high-priority strata (eg, 80-89 and 90+), survival on the waiting list is poor in the absence of transplant, with few candidates $(<10 \%)$ alive on the waiting list at 90 days. Net survival benefit in the high-priority strata was also minimal as a result of their relatively poor posttransplant survival. These findings suggest that additional modifications to the current allocation system must be considered.

The authors thank the UNOS for supplying data and Katarina Anderson for assistance with our analysis.

\section{References}

1. Egan TM, Murray S, Bustami RT, et al. Development of a new lung allocation system in the United States. Am J Transplant. 2006;2:1212-27.

2. Pierson $\mathrm{RN}, \mathrm{Br} \mathrm{ML}, \mathrm{McCullough} \mathrm{KP}$, et al. Thoracic organ transplantation. Am J Transplant. 2004;4:93-105.

3. Travaline JM, Cordova FC, Criner GJ, Furukawa S, Criner GJ. Discrepancy between severity of lung impairment and seniority on the lung transplantation list. Transplant Proc. 2004;36:3156-60.

4. Department of Health and Human Services. Organ Procurement and Transplantation Network: final rule; 42 CFR-Part 121. Federal Register. October 20, 1999;56649-61.

5. Kozower BD, Meyers BF, Smith MA, et al. The impact of the lung allocation score on short-term transplantation outcomes: a multicenter study. J Thorac Cardiovasc Surg. 2008;135:166-71.

6. Osaki S, Maloney JD, Meyer K, et al. The impact of the lung allocation scoring system at the single national Veterans Affairs Hospital lung transplantation program. Eur J Cardiothorac Surg. 2009;36:497-501.

7. Merlo CA, Weiss ES, Orens JB, et al. Impact of U.S. lung allocation score on survival after lung transplantation. J Heart Lung Transplant. 2009;28 769-75.

8. Gries CJ, Mulligan MS, Edelman JD, et al. Lung allocation score for lung transplantation. impact on disease severity and survival. Chest. 2007;132:1954-61.

9. Iribarne A, Russo MJ, Davies RR, et al. Despite decreased wait list times for lung transplantation, lung allocation scores continue to increase. Chest. 2009;135: 923-8.

10. McCue JD, Mooney J, Quail J, et al. Ninety-day mortality and major complications are not affected by use of lung allocation score. J Heart Lung Transplant. 2008;27:192-6.

11. Liu V, Zamora MR, Dhillon GS, Weill D. Increasing lung allocation scores predict worsened survival among lung transplant recipients. Am J Transplant. 2010 10:1-6.

12. Russo MJ, Iribarne A, Hong KN, et al. High lung allocation score is associated with increased morbidity and mortality following transplantation. Chest. 2010; 137:651-7.

13. Egan TM, Kotloff RM. Pro/Con debate. Chest. 2005;128:407-15. 
14. Davis SQ, Garrity ER. Organ allocation in lung transplant. Chest. 2007;132: 1646-51.

15. Russo MJ, Davies RR, Hong KN, et al. Who is the high-risk recipient? Predicting mortality after lung transplantation using pretransplant risk factors. $J$ Thorac Cardiovasc Surg. 2009;138:1234-8.

16. Gries CJ, Rue TC, Heagerty PJ, Edelman JD, Mulligan MS, Goss CH. Development of a predictive model for long-term survival after lung transplantation and implications for the lung allocation score. J Heart Lung Transplant. 2010;29: $731-8$

\section{APPENDIX 1.}

Calculation of the LAS involves the following patient characteristics: age, body mass index, diabetes, functional status, forced vital capacity ( $\%$ predicted), systolic pulmonary artery pressure, pulmonary capillary wedge pressure, oxygen requirement, 6-minute walk distance less than $150 \mathrm{ft}$, continuous mechanical ventilation, partial pressure of $\mathrm{CO}_{2}$, diagnosis, and creatinine.

The derivation of the LAS algorithm is explained in further detail at the following UNOS website: http://www. unos.org/docs/lung_allocation_score.pdf.

\section{Discussion}

Dr Michael Smith (Phoeniz, Ariz). Your work addresses an important question given the ongoing difficult problems with scarcity of resources in transplantation principle, that is, the donor organs themselves and the economic constraints on health care in general, particularly in the arena of lung transplantation. The University of Washington group and others (eg, the group in The Netherlands) recently showed that among other common solid-organ transplants, lung transplantation has been demonstrated to be the most expensive, and this is mainly because of the higher cost of postrecovery care. Many have suggested that although lung transplantation can substantially improve quality of life, it has limited cost-effectiveness. As a reaction to the difficult economic environment that we face today, some payers, including the Medicaid system of my home state of Arizona, have determined that given the limited survival advantages and poor cost-effectiveness, they will no longer cover lung transplantation for their insured lives over the age of 21 years. Given that we know we are trying to do the greatest good for our patients with limited resources, how do we as transplant physicians and surgeons act as good stewards of these limited resources so that we can continue to provide care to our patients? That is just a philosophical question.

The LAS has been an important step in the right direction in allowing us to direct these limited resources more appropriately compared with the old system of directing them simply by time on the waiting list. Work such as yours helps to identify continued limitations in the current system and may help to point out facets of the system that perhaps can be improved.

Given your findings, I have just a couple other comments and questions. Your findings seem to identify some important limitations of the current LAS system. One is that in approximately $90 \%$ of cases, our resources are going to patients who we are not offering a survival benefit to and those in the lowest priority group are actually better off without transplant from a survival standpoint. At the other end of the spectrum, in the higher- priority groups, the LAS seems to over-advantage, if you will, the sickness of the patient over the likelihood of an improved survival after transplantation. As you mentioned, more longterm data need to assessed to be certain of some of these conclusions, but given what you have at the present time, what changes to the current LAS system would you recommend to address these problems at either end of the LAS stratification groups?

Dr Russo. Those are great questions. First, this analysis is not meant to represent a criticism of the LAS. The LAS is a great step forward over the previous system, which was essentially based on waiting time alone. But under the current LAS system, issues remain. The system should be designed to maximize the net benefit of transplant However, medical urgency is weighted more heavily than posttransplant survival. Therefore, patients in the highest priority groups are sickest at the time of transplant and thus, as expected, have the worst posttransplant outcomes. This may prevent our maximizing the benefits from available organs. In the future, I would support exploring the equal weighting of expected survival on the waiting list and posttransplant survival in future iterations of LAS. Second, as you point out, approximately $90 \%$ of the patients receiving transplants had lower-priority LAS scores $(<50)$. Concurrently, there were approximately 350 patients in the higher-priority groups who died on the waiting list over the study period. You have to wonder why we're performing transplantations in patients in the lower-priority group when there are patients in the higher-priority group who die on the waiting list. It may be because those patients were not appropriate for transplant or for the donors who are available during their listing time. However, I do not think this is the primary explanation.

Dr Smith. In terms of your methods, you looked at graft survival rather than patient survival after transplantation, and that begs the question to me, do you think that re-transplantation plays a significant role in improving survival in the groups of patients receiving transplants or offered transplantation, and could this bias your data against a survival benefit of transplantation in the end?

Dr Russo. Graft survival is the standard measure of survival in our analysis. In our opinion, graft survival has a number of advantages over patient survival. First, given the critical scarcity of organs available for transplantation, further expanding the benefit of transplantation is predicated on improving the use of organs available for transplantation. Analysis focusing on graft survival rather than patient survival better addresses this important issue. Moreover, if recipients receive multiple transplants during the study period, survival obtained after retransplantation may be double counted. That is, patient survival for recipients undergoing transplantation twice during the study period is the sum of survival of the initial transplant plus the survival after the second transplant; patient survival after the second transplant will also be included in analysis as a second, distinct observation, and thus is counted twice. Finally, using patient survival in regression analysis significantly confounds the model by allowing for a bailout strategy of retransplant and introduces greater confounding by leaving factors unaccounted for in the model, including the timing of the retransplant, recipient clinical status at the time of the repeat transplant, and characteristics of the donor involved in the retransplant.

Dr Smith. In the big picture, we cannot ignore the improvements in quality of life that are offered to many of these 
patients because many would argue that lung transplantation does allow many of our patients to become productive economically, both socially and economically, for their families and society at large. I know that quality of life is beyond the scope of this article, but perhaps you could comment on the relative importance in these different patient priority groups, especially in the absence of a demonstrated survival benefit. Along these lines, how much should we weight quality of life in comparison with survival advantage as we go forward in trying to improve the LAS system?

Dr Russo. This is an excellent question. UNOS is a great resource because there are comprehensive and longitudinal survival data. In outcome research, we frequently say that death is the ultimate end point. But it is increasingly being recognized that quality of life may be equally or more important. I think this is certainly the case here.

Although the UNOS provides functional status data on recipients posttransplant, it is generally poorly populated, and there are no longitudinal quality of life data on waiting list candidates. Findings from this analysis highlight the importance of these data, especially given the finding of limited survival benefit in the lower-priority strata. It is possible that despite the limited survival benefit in some subgroups, there may be a significant benefit in terms of quality-adjusted life years.

Therefore, a national effort should begin to measure quality of life in candidates and recipients alike. Furthermore, future studies should assess candidates' preferences, including their willingness to trade long-term survival for improved quality of life. This may be particularly relevant to candidates in the lower-priority strata who have longer survival on the waiting list, but poor quality of life.

As stated in the Presidential Address, we need to be the stewards of this effort. Given payors' increasing resistance to reimburse for costly therapies, if we cannot demonstrate a benefit from the therapies that we offer, going forward, we may not be allowed to provide them.

Dr Michael Mulligan (Seattle, Wash). Congratulations on changing your title from when it was submitted to when it was presented. I think the title as it reads is a bit misleading, and for those of us who sit on the pulmonary council, it is scary because the LAS offers no guidance as to who should not receive a transplant. Our charge over the next several years is to try to help refine that, with help from our European colleagues.

I want to invite you to opine about 2 potential issues. One is if you are assuming that limited survival benefit and a difficult to quantitate quality of life benefit from performing transplantation in a candidate with LAS less than 40, what would you propose? Broader regional sharing akin to what we do for heart transplants, where it is local $1 \mathrm{~A}$ and $1 \mathrm{~B}$, and then it is farmed out before you come back to a local status 2? And the other is because we double weight waitlist mortality in calculating the LAS, what should we do when the LAS starts to crest over 80 ? Because it is not a selfcorrecting program, what would you propose for providing wider more general usable user-friendly guidance for who should not undergo transplantation?

Dr Russo. Those are great questions. First in terms of the organ allocation issue, this is a question that our group is interested in exploring. We have preliminary data on this, which I did not present here. However, if you look at the patients who undergo transplantation with LAS less than 40, not surprisingly most of those organs are procured locally, whereas candidates with LAS greater than 80 , organs were much more likely to be procured regionally or nationally. This raises questions about broader organ sharing because there be minimal benefit in performing transplantation in the lower-priority group; in fact, there may be a negative benefit, and again we have patients in the higher-priority groups dying on the waitlist. Therefore, my bias is that, yes, there should be broader organ sharing. However, the first step is to establish why low-priority candidates are undergoing transplantation at such a high rate while higher-priority candidates are dying on the waitlist.

Regarding your second question, and I agree it is an important issue, how should we weigh medical urgency versus expected posttransplant survival? I cannot prove this, but I think that more heavily weighing medical urgency prevents us from maximizing benefit. 